\title{
An NMRA-Like Protein Regulates Gene Expression in Phytophthora capsici to Drive the Infection Cycle on Tomato
}

\author{
Jasmine Pham, ${ }^{1,2}$ Remco Stam, ${ }^{3}$ Victor Martinez Heredia, ${ }^{1}$ Michael Csukai, ${ }^{4}$ and Edgar Huitema ${ }^{1,2,+}$ \\ ${ }^{1}$ Division of Plant Sciences, University of Dundee, Dundee DD2 5DA, U.K.; ${ }^{2}$ Dundee Effector Consortium, James Hutton \\ Institute, Invergowrie, Dundee DD2 5DA, U.K.; ${ }^{3}$ School for Life Sciences, Weihenstephan Technische Universität München, \\ Freising, Germany; and ${ }^{4}$ Syngenta, Jealott's Hill International Research Centre, Bracknell, U.K.
}

Accepted 6 February 2018.

\begin{abstract}
Phytophthora spp. cause devastating disease epidemics on important crop plants and pose a grave threat to global crop production. Critically, Phytophthora pathogens represent a distinct evolutionary lineage in which pathogenicity has been acquired independently. Therefore, there is an urgent need to understand and disrupt the processes that drive infection if we aspire to defeat oomycete pathogens in the field. One area that has received little attention thus far in this respect is the regulation of Phytophthora gene expression during infection. Here, we characterize PcNMRAL1 (Phyca11_505845), a homolog of the Aspergillus nidulans nitrogen metabolite repression regulator NMRA and demonstrate a role for this protein in progression of the Phytophthora capsici infection cycle. PcNmrAL1 is coexpressed with the biotrophic marker gene PcHmpl (haustorial membrane protein 1) and, when overexpressed, extends the biotrophic infection stage. Microarray analyses revealed that $P c N m r A L 1$ overexpression in $P$. capsici leads to large-scale transcriptional changes during infection and in vitro. Importantly, detailed analysis reveals that $P c N m r A L 1$ overexpression induces biotrophy-associated genes while repressing those associated with necrotrophy. In addition to factors controlling transcription, translation, and nitrogen metabolism, PcNMRAL1 helps regulate the expression of a considerable effector repertoire in $P$. capsici. Our data suggests that PcNMRAL1 is a transcriptional regulator that mediates the biotrophy to necrotrophy transition. PcNMRAL1 represents a novel factor that may drive the Phytophthora disease cycle on crops. This study provides the first insight into mechanisms that regulate infection-related processes in Phytophthora spp. and provides a platform for further studies aimed at disabling pathogenesis and preventing crop losses.
\end{abstract}

The microarray data generated during the current study are available in the ArrayExpress depository under accession E-MTAB-5620 and the experimental design and probe sequences under A-MTAB-610.

${ }^{\dagger}$ Corresponding author: Edgar Huitema; E-mail: E.Huitema@dundee.ac.uk

Funding: This study is funded by the Biotechnology and Biological Sciences Research Council grant BB/J017817/1.

*The $\boldsymbol{e}$-Xtra logo stands for "electronic extra" and indicates that six supplementary figures, seven supplementary tables, and four supplementary files are published online.

(c) 2018 The American Phytopathological Society
Plant-pathogenic oomycetes cause devastating diseases on a wide range of plants important in food production, forestry, and natural ecosystems. For example, Phytophthora infestans, the causal agent of late blight on potato and tomato, continues to cause multibillion dollar losses each year (Birch and Whisson 2001; Nowicki et al. 2012). Other economically devastating pathogens in the genus include $P$. sojae and $P$. capsici, the major disease-causing agents of soybean and pepper, respectively. The vast economic damage that Phytophthora pathogens cause have driven and continue to drive efforts to understand the basic processes involved in pathogenicity (Kamoun et al. 2015).

Detailed phenotypic and cytological studies have greatly aided our understanding the biology of Phytophthora diseases. Generally, the Phytophthora infection cycle starts with the formation of penetration hyphae from motile spores (zoospores) that have encysted or from sporangia. Upon ingress of susceptible host tissue, Phytophthora hyphae first grow and colonize the apoplast while extending protrusions (haustoria) that breach the cell-wall barrier and give rise to an intimate interface with the plant cell membrane that is thought to facilitate extensive signaling exchanges and maintain the biotrophic infection phase (Mendgen and Hahn 2002). Despite pathogen ingress, a lack of plant cell death is evident during this early stage, indicating the presence of mechanisms that, on the one hand, allow suppression of host defense signaling cascades while, on the other hand, tightly control developmental processes associated with disease progression. Indeed, comparisons between multiple genome sequences, those from Phytophthora in particular, have provided a blueprint of the components that are generally required for disease and development (Haas et al. 2009; Lamour et al. 2012a; Pais et al. 2013). Following biotrophy, Phytophthora infection features a distinct necrotrophic phase that is marked by death and tissue collapse, culminating in the production of sporangia, which, on dispersal, initiate a new infection cycle (Birch and Whisson 2001; Lamour et al. 2012b; Tyler 2007). Although infection on the phenotypic level has been well described, the molecular mechanisms driving disease development in this important group of pathogens is yet elusive.

Previously, we have defined and described dramatic changes in $P$. capsici gene expression associated with its distinct infection stages, the biotrophic phase from 0 to $24 \mathrm{~h}$ postinoculation (hpi), when few phenotypic symptoms are visible, and the necrotrophic phase from 24 hpi onward, resulting in cell death and tissue collapse (Jupe et al. 2013). Detailed microarray analyses of a tomato infection time course revealed that a large set of differentially expressed genes (DEGs) feature stage-specific changes in transcript levels, possibly underpinning hemibiotrophy in $P$. capsici. An analysis of stage-specific gene expression helped 
identify a suite of 57 P. capsici genes on the basis of coexpression with PcHmpl (P. capsici haustorial membrane protein 1), a homolog of the well-defined marker of biotrophy in $P$. infestans PiHmpl, and 209 genes associated with the onset of necrotrophy, as defined by coexpression with PcNppl (Avrova et al. 2008). This and other studies detailing Phytophthora gene-expression analyses have led to the view that gene regulation is tightly controlled and required for successful infection and disease progression. Critically, despite the importance of regulation, little is known about the factors or mechanisms that drive stage-specific gene expression during infection and how this is linked to disease progression on host plants.

We hypothesized that transcriptional regulators (TRs), which specify gene expression during biotrophy, are coexpressed with PcHmpl and help regulate the infection cycle. To test this hypothesis, we selected and characterized an NMRA (nitrogen metabolic regulation A)-like protein-coding gene (referred to as PcNmrAL1 from here on) as a candidate TR that is coexpressed with PcHmpl.

The role of NMRA-like proteins in gene regulation has been well-studied in fungi (Andrianopoulos et al. 1998; Dunn-Coleman et al. 1981; Lamb et al. 2004, 2003; Stammers et al. 2001; Tomsett et al. 1981). Given the role of fungal NMRA-like proteins in transcriptional regulation, the link with pathogenicity and virulence in fungal plant pathogens, and the apparent biotrophyassociated expression of PcNmrAL1, we chose to further investigate PcNMRAL1 function in $P$. capsici. In particular, we asked whether PcNMRAL1 acts as a TR during the biotrophic infection phase.
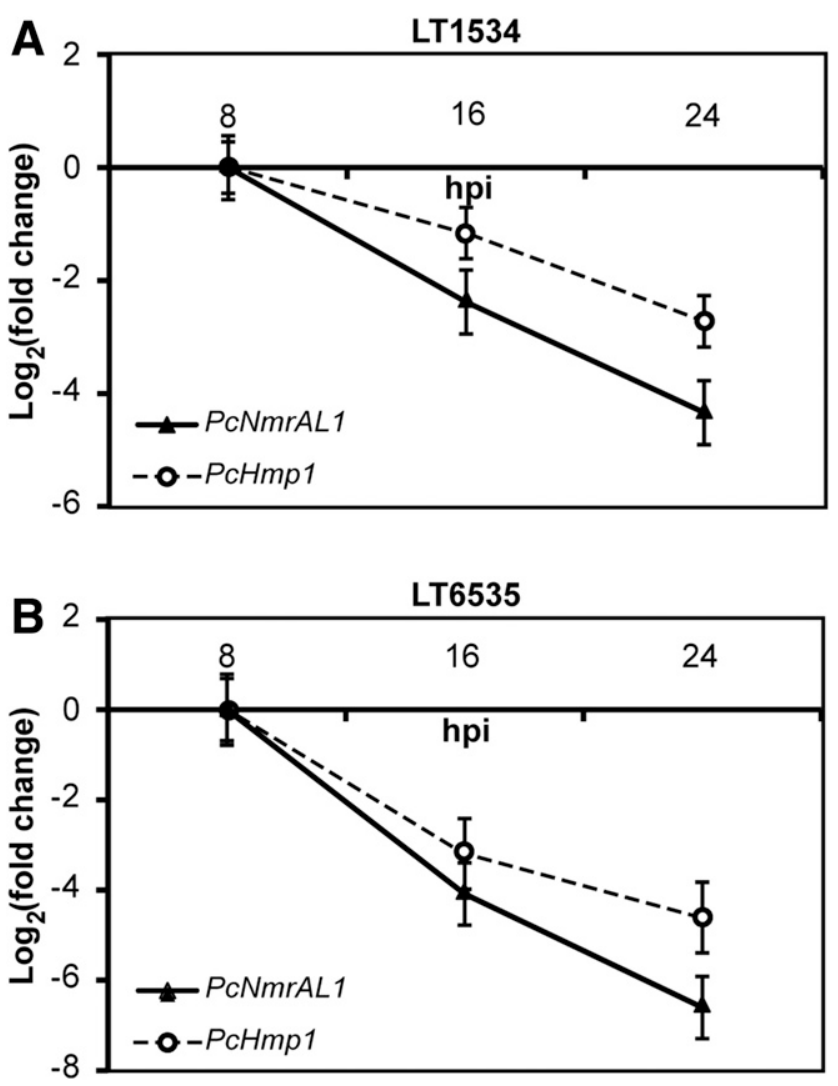

Fig. 1. Endogenous $P c N m r A L 1$ and $P c H m p l$ are coexpressed during early infection. Gene expression levels of PcNmrALl and PcHmpl in the Phytophthora capsici strains A, LT1534 and B, LT6535 on infection of tomato leaves over a time-course infection assay were measured by quantitative reverse transcription-polymerase chain reaction and are shown relative to the $8 \mathrm{~h}$ postinoculation (hpi) timepoint. Data shown is the average of four biological replicates in the LT1534 background and six biological replicates in the LT6535 background. Error bars represent standard error of the means.
Overexpression of PcNmrALl coupled with virulence assays, microarray analysis of a time course infection assay, and gene ontology (GO) analysis of DEGs was used to investigate the role of PcNMRAL1 in regulating gene expression in $P$. capsici during infection of host tomato leaves. Infection assays using strains overexpressing PcNmrALl showed a negative impact on visible lesion growth. Detailed gene expression analyses subsequently helped establish de-regulation of both biotrophy and necrotrophyassociated gene expression, suggesting a dual role for PcNMRAL1 in the regulation of gene expression and disease progression. Further interrogation of genes found to be differentially expressed in the PcNmrAL1-overexpressing line compared with a control line was used to link altered expression of transcription factors (TFs), TRs, and effector proteins, as well as genes involved in nitrogen metabolism to the changes in gene expression and virulence we observed. Taken together, our results suggest that PcNMRAL1 mediates the biotrophy to necrotrophy transition and, thus, represents a novel factor that may drive the Phytophthora disease cycle on crops. This study provides a first insight into the mechanisms that regulate infection-related processes in Phytophthora spp. and provides a platform for further studies aimed at disabling pathogenesis and preventing crop losses.

\section{RESULTS}

\section{PcNmrAL1 is expressed during biotrophy.}

Previous work in our lab identified a set of 57 P. capsici genes for which expression was correlated with PcHmpl (Jupe et al. 2013). Within this list of 57 genes, we identified a candidate TR (Phyca11_505845) annotated as containing an NAD(P)-binding/ NmrA-like domain ( $P$. capsici LT1534 v11.0, PFAM domain PF05368, InterPro domains IPR008030 and IPR016040). Querying the PcNMRAL1 sequence against the ProDom protein domain family database (release 2012.1) also gave matches to NMRA-like family ProDom domains (Supplementary Table S1). In Aspergillus nidulans, the NMRA protein (AnNMRA) has previously been well-characterized and has been shown to act as a TR of nitrogen metabolism. Sequence analyses revealed $22.4 \%$ identity, and $33.3 \%$ similarity of PcNMRAL1 to AnNMRA, while BLASTP and TBLASTN searches of PcNMRAL1 against proteins of other Phytophthora species revealed the presence of similar NMRA-like proteins (Supplementary Table S2).

To confirm expression of PcNmrALl during biotrophy, we measured the expression of both PcNmrALI and PcHmpl across an infection time course. For this purpose, inoculation experiments were performed with two wild-type $P$. capsici strains, LT1534 and LT6535, and tissue was harvested for RNA extraction at 8, 16, and 24 hpi. Quantitative reverse transcription-polymerase chain reaction (qRT-PCR) revealed that, at the timepoints measured, levels of $P c N m r A L 1$ were highest $8 \mathrm{hpi}$ and were lower (relative to $8 \mathrm{hpi}$ ) at 16 and 24 hpi (Fig. 1A and B). Consistent with biotrophyassociated expression of PcNmrAL1, PcHmpl featured highly similar gene expression patterns, suggestive of coregulation in the biotrophic phase (Fig. 1A and B). Furthermore, gene expression patterns of PcNmrAL1 and PcHmpl were similar in both LT1534 and LT6535, demonstrating that our observations are both reproducible and isolate-independent. Comparison of PcNmrAL1 expression levels between mycelia grown in vitro and the early infection stages on tomato confirmed induction of PcNmrAL1 expression during biotrophy (Supplementary Fig. S1). Taken together, our results firmly demonstrate biotrophy-associated expression of $P c N m r A L 1$, implying a role in infection.

\section{Overexpression of $\mathrm{PcNmrAL1}$ affects \\ lesion development in planta.}

We hypothesized that overexpression of PcNmrAL1 would lead to misregulation of genes normally required for disease, which, if 
true, could result in an altered infection phenotype. To test this hypothesis, we transformed strain LT6535 with a construct carrying a FLAG-tagged version of PcNmrAL1 (pTOR::FLAGPcNMRAL1). An equivalent construct carrying tdTomato instead of PcNmrAL1 (pTOR::tdTom) was also used to generate transformants that could serve as control lines. We tested multiple independent transformants for PcNmrAL1 overexpression by means of qRT-PCR and Western blots. Transformants featuring the highest level of ectopic PcNmrAL1 expression were chosen for follow-up experiments (Supplementary Fig. S2). To determine if overexpression of PcNmrAL1 affected virulence on tomato, we drop-inoculated detached tomato leaves with spore suspensions of wild type and pTOR::tdTom and pTOR::FLAG-PcNMRAL1 transformants. Lesion development was assessed at 1,2, and 3 days postinoculation (dpi). Measurement of $P$. capsici disease progression revealed a significant reduction in lesion growth for our transformant overexpressing $P c N m r A L 1$ when compared with the tdTomato counterpart and wild-type strain (Fig. 2). In leaves inoculated with $P$. capsici overexpressing PcNmrAL1, lesions did not spread beyond the inoculation droplet at $24 \mathrm{hpi}$, in contrast to lesions caused by wild-type $P$. capsici and strains overexpressing tdTomato (Fig. 2A). Although lesions caused by our pTOR:: FLAG-PcNMRAL1 line did increase in size over time, they remained significantly smaller than those caused by wild-type or the pTOR::tdTom strains throughout the duration of the experiment (Fig. 2B). Critically, equivalent experiments in the LT1534 background gave similar results, suggesting that the observed PcNmrAL1 overexpression phenotype is robust and strain independent (Supplementary Fig. S3). We assessed growth rates of both our wild-type and transformed strains in vitro and found marginal yet consistent reduction in colony expansion of the PcNmrALl overexpression line in comparison with control lines
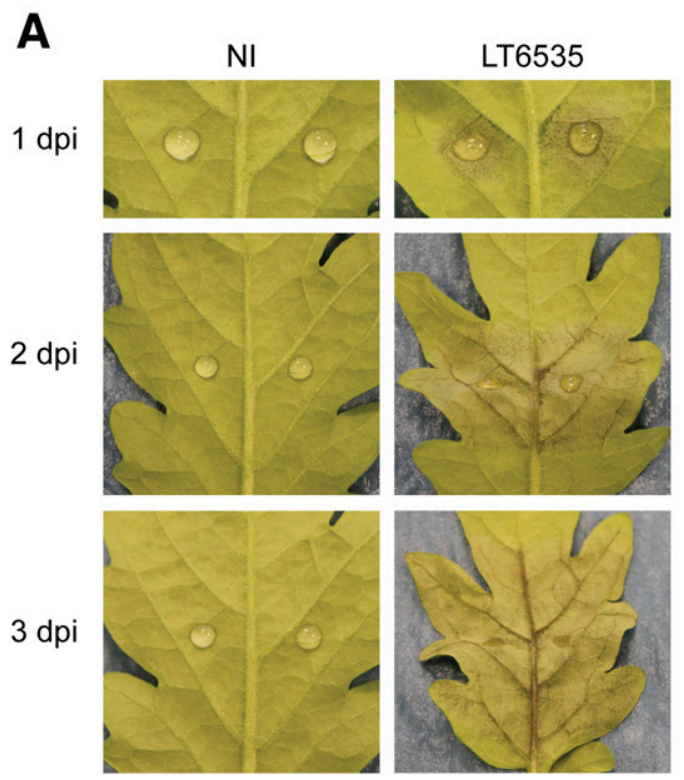

B

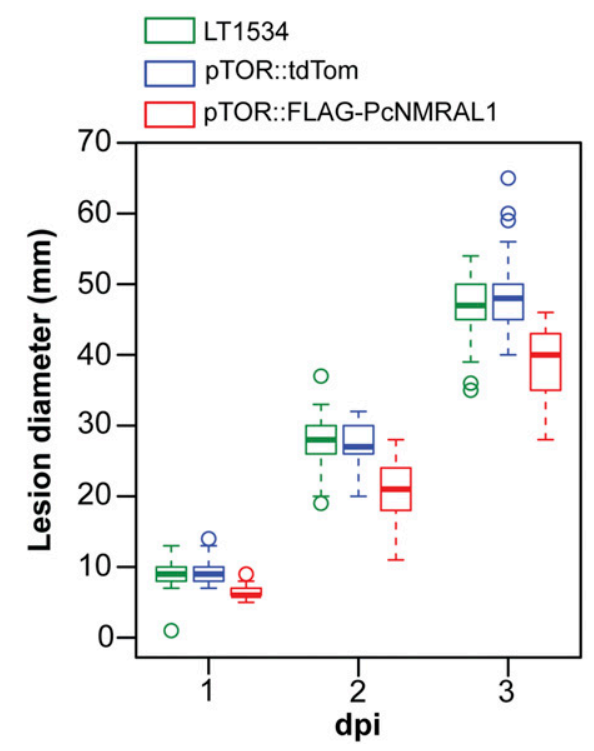

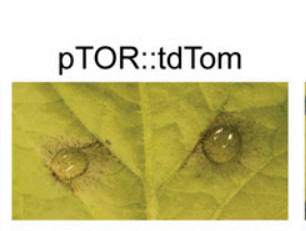

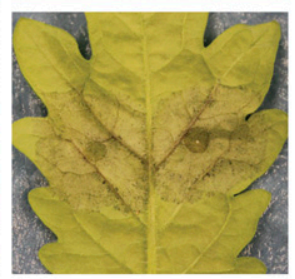

PTOR::FLAG-
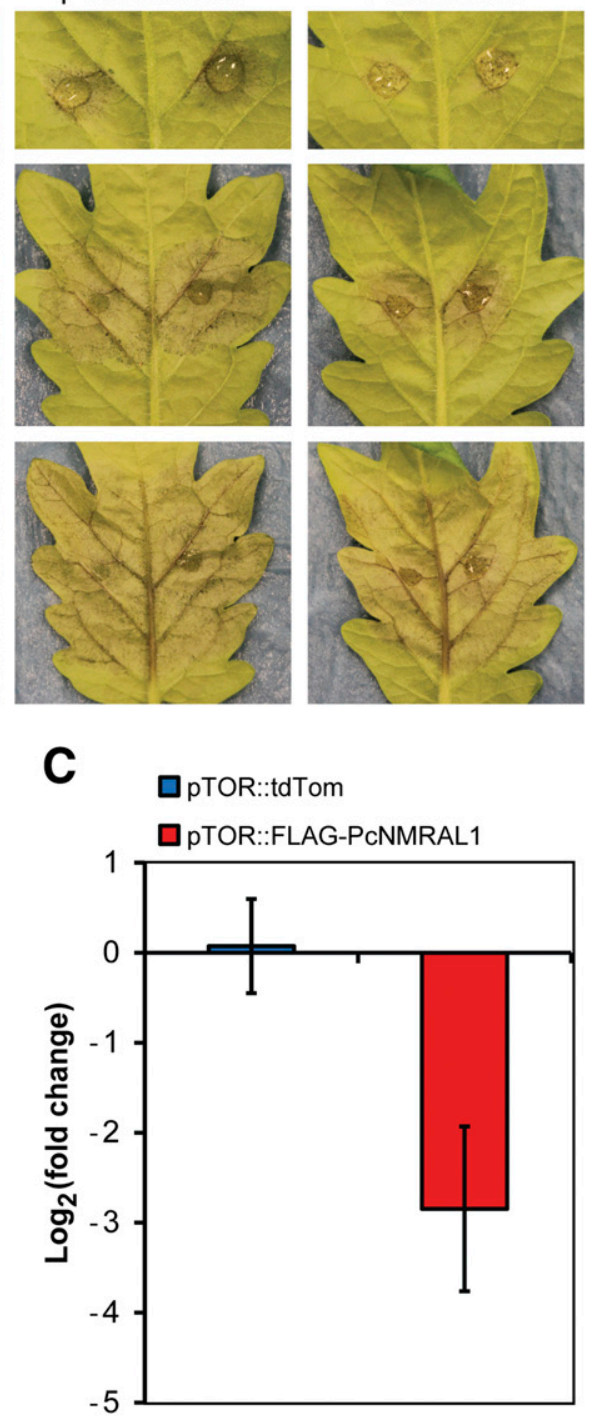

Fig. 2. Overexpression of PcNmrAL1 in Phytophthora capsici LT6535 results in reduced symptom development on tomato leaves. A, Lesion appearance over a time-course infection assay. Leaves were photographed at 1, 2, and 3 days postinoculation (dpi). NI = noninfected/water. B, Lesion diameters measured differed significantly between the pTOR::FLAG-PcNMRAL1 line with either the wild-type or pTOR::tdTom line at $P<0.0001)$. Differences in lesion diameter between the wild-type and pTOR::tdTom lines were less significant $(P=0.969)$. Data shown are from three replicate experiments consisting of a total of 90 lesion measurements for each treatment. C, Overexpression of $P c N m r A L 1$ also results in reduced biomass in planta at $16 \mathrm{~h}$ postinoculation. Quantification of $P$. capsici biomass was by quantitative polymerase chain reaction targeting $P$. capsici tubulin (Phyca11_576734) and normalized against tomato tubulin (Solyc08g006890.2.1). Values shown are relative to that found in wild-type $P$. capsici in planta, represented by $\log _{2}$ (fold change) $=0$. Data shown are from six biological replicates consisting of three leaves for each replicate, for each line. 
(wild-type and pTOR::tdTom strains) (Supplementary Fig. S4). For this reason, we used qPCR to assess the level of $P$. capsici biomass at $16 \mathrm{hpi}$ in infected tomato leaf tissue. Comparisons between our pTOR::tdTom and pTOR::FLAG-PcNMRAL1 lines revealed a dramatic eightfold reduction in biomass for our PcNmrAL1 overexpression line (Fig. 2C), indicative of significant impairment of $P$. capsici growth in the early stages of infection.

\section{Overexpression of $\mathrm{PcNmrAL1}$ prolongs the expression of the biotrophy marker gene $\mathrm{PcHmp1I}$.}

As PcNmrAL1 is coexpressed with $\mathrm{PcHmpl}$ during infection in wild-type $P$. capsici strains, we wanted to determine whether overexpression of PcNmrALl affects PcHmpl expression or regulation. To investigate this, we measured PcNmrALl and PcHmpl expression in pTOR::tdTom and pTOR::FLAGPcNMRAL1 lines, during infection at 8,16 , and $24 \mathrm{hpi}$, by qRT-PCR and compared these to levels found in wild-type LT6535. This analysis confirmed overexpression of PcNmrAL1 in our pTOR::FLAG-PcNMRAL1 line as transcript levels were found to be elevated at every timepoint measured, when compared with wild type (Fig. 3A). Overexpression was specific

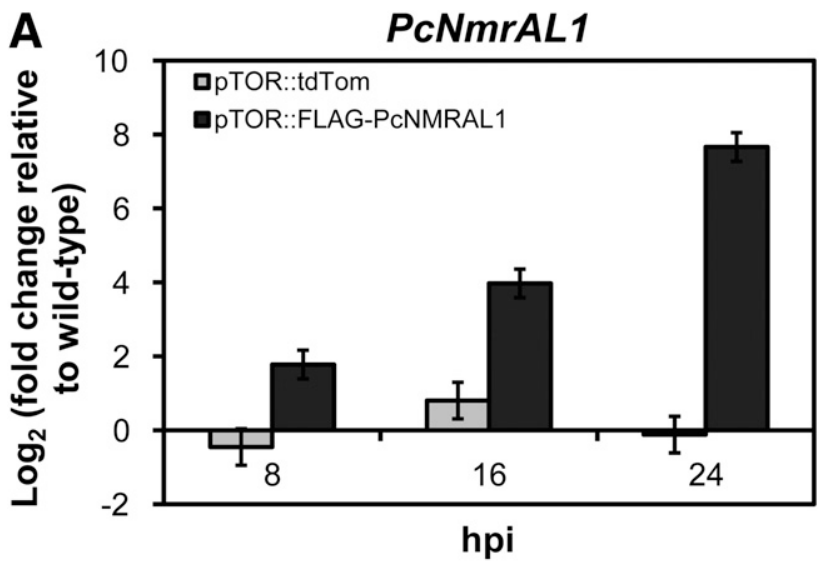

B

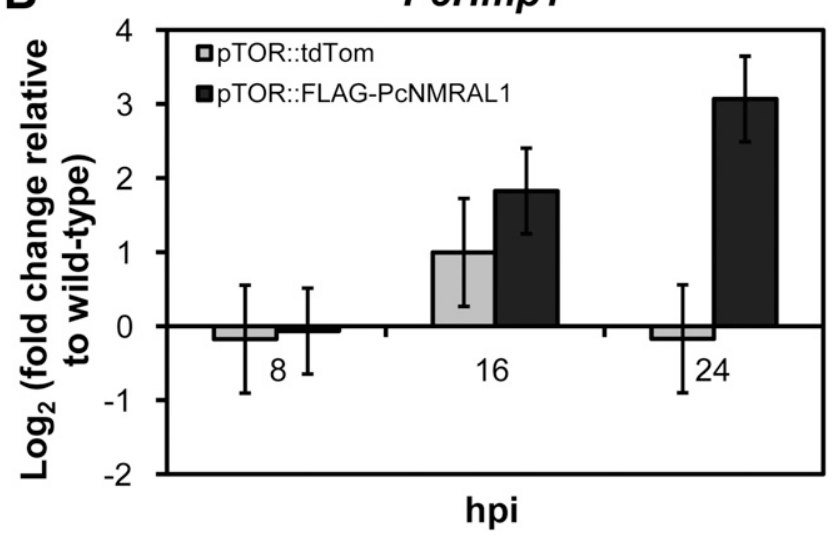

Fig. 3. Overexpression of $P c N m r A L 1$ prolongs expression of PcHmpl. A, Expression of PcNmrAL1 and $\mathbf{B}$, PcHmpl in pTOR::tdTom and pTOR:: FLAG-PcNMRAL1 lines in the LT6535 background in planta during infection of tomato leaves, measured by quantitative reverse transcriptionpolymerase chain reaction. Expression levels shown are relative to wildtype LT6535, represented by $\log _{2}$ (fold change) $=0$. PcNmrAL1 levels were significantly different between pTOR::tdTom and pTOR::FLAGPcNMRAL1 ( 8 h postinoculation [hpi]: $P<0.01,16$ hpi: $P<0.001$, and 24 hpi: $P<0.0001)$. PcHmpl levels were significantly different between pTOR::tdTom and pTOR::FLAG-PcNMRAL1 at 24 hpi $(P<0.0001)$. Error bars represent standard error of the means. Data shown is the average of six biological replicates consisting of three leaves for each replicate, for each line. and not due to the transformation protocol, as PcNmrAL1 expression levels in the pTOR::tdTom line closely resembled those found in our wild-type control (Fig. 3A). We also found evidence for elevated levels of PcHmpl transcripts attributable to PcNmrAL1 overexpression; specifically, levels of PcHmpl expression were significantly enhanced at 24 hpi when compared with the wild type, in contrast to results obtained with the control pTOR::tdTom line (Fig. 3B). Collectively, these results suggest that overexpression of PcNmrAL1 results in the maintenance of high PcHmpl expression levels in the later infection stages, when abundance of endogenous levels of both PcNmrAL1 and PcHmpl transcripts normally decrease. This maintenance of $\mathrm{PcHmpl}$ levels coupled with the reduced lesion development on infection may indicate a prolonged biotrophic phase in the PcNmrALl overexpression line. Given the effect of overexpression of PcNmrALI on PcHmpl expression and disease progression, we hypothesized that PcNmrAL1 contributes to the regulation of other infection-related genes.

\section{Overexpression of $\mathrm{PcNmrAL1}$ affects expression of a large set of genes in $P$. capsici during infection.}

To determine the effect of PcNmrALl overexpression on gene expression in $P$. capsici, a microarray experiment of a time course infection over 8,16 , and 24 hpi was performed with the pTOR::FLAG-PcNMRAL1 overexpression strain in the LT6535 background. In this experiment, our LT6535 isolate transformed with pTOR::tdTom was used as a control. The arrays contained $P$. capsici-specific probes allowing us to measure the levels of 20,077 transcripts. Statistical analyses aimed at identifying DEGs during the time course in each strain identified 9,035 and 7,296 genes in our pTOR::tdTom and pTOR::FLAGPcNMRAL1 datasets, respectively, confirming extensive transcriptional changes during infection (Supplementary Fig. S5). Critically, there was significant overlap between our sets as 6,496 genes were differentially expressed in both the pTOR::tdTom and pTOR::FLAG-PcNMRAL1 lines. Conversely, 2,539 and 800 genes were shown to significantly change only in the pTOR:: tdTom line or the pTOR::FLAG-PcNMRAL1 line, respectively. These results indicate that i) large-scale changes in gene expression across our time course are broadly similar between strains and ii) there may be a significant set of genes for which expression changes as a consequence of PcNmrAL1 overexpression.

To identify genes that have altered expression due to elevated PcNMRAL1 levels, we performed DEG analyses between our strains at the 8-, 16-, and 24-hpi timepoints. These analyses revealed genes that were differentially expressed, possibly because of PcNmrAL1 overexpression. Consistent with the observation that endogenous PcNmrALl levels are high in the earliest stages of infection (at which the impact of overexpression is smallest) and drops subsequently, DEG analyses at each timepoint revealed a growing impact of PcNmrAL1 overexpression on the $P$. capsici transcriptome as infection progressed to the later stages. A total of 915 genes were affected by PcNmrALl overexpression at 8 hpi compared with 5,735 and 4,795 at 16 and 24 hpi, respectively (Fig. 4A; Supplementary File S1). Interestingly, PcNmrAL1 overexpression resulted in both the induction and repression of $P$. capsici gene expression, suggesting a complex role for this protein in modulating the transcriptome.

Within the genes identified as DEGs in the microarray data were PcNmrALl and PcHmpl, as expected, as well as two other marker genes, PcCdc14 (Phyca11_510939) and PcNpp1 (Phyca11_11951). From the microarray data, expression of PcNmrALl and PcHmpl was higher at 16 and 24 hpi in the PcNmrAL1 overexpression line (Fig. 4B and C), in agreement with previous qRT-PCR data (Fig. 3). $\mathrm{PcCdc14}$ encodes for a 
phosphatase that is expressed during sporulation and for a short time after germination (Ah Fong and Judelson 2003), whereas PcNppl (Nepl-like protein 1) is expressed during the later necrotrophic phase (Ah Fong and Judelson 2003; Kanneganti et al. 2006). In the pTOR::tdTom line, expression of these two marker genes followed the expected patterns, with $\mathrm{PcCdc14}$ levels decreasing from 8 to $16 \mathrm{hpi}$ and, then, increasing again between 16 to 24 hpi, whereas PcNppl levels increase over time as infection progresses (Fig. 4D and E; Supplementary Fig. S6A and B). In comparison, in the pTOR::FLAG-PcNMRAL1 line, $P c C d c 14$ levels continue to decrease over the time course and PcNppl levels, although increasing over time as seen in the pTOR::tdTom line, increase at a slower rate (Fig. 4D and E). The prolonged expression of PcHmpl coupled with the lower expression of $P c C d c 14$ and $P c N p p l$ during the later stages of biotrophy suggests overexpression of PcNmrALl affects the

A

\section{Repressed by PcNMRAL1}

Induced by PcNMRAL1

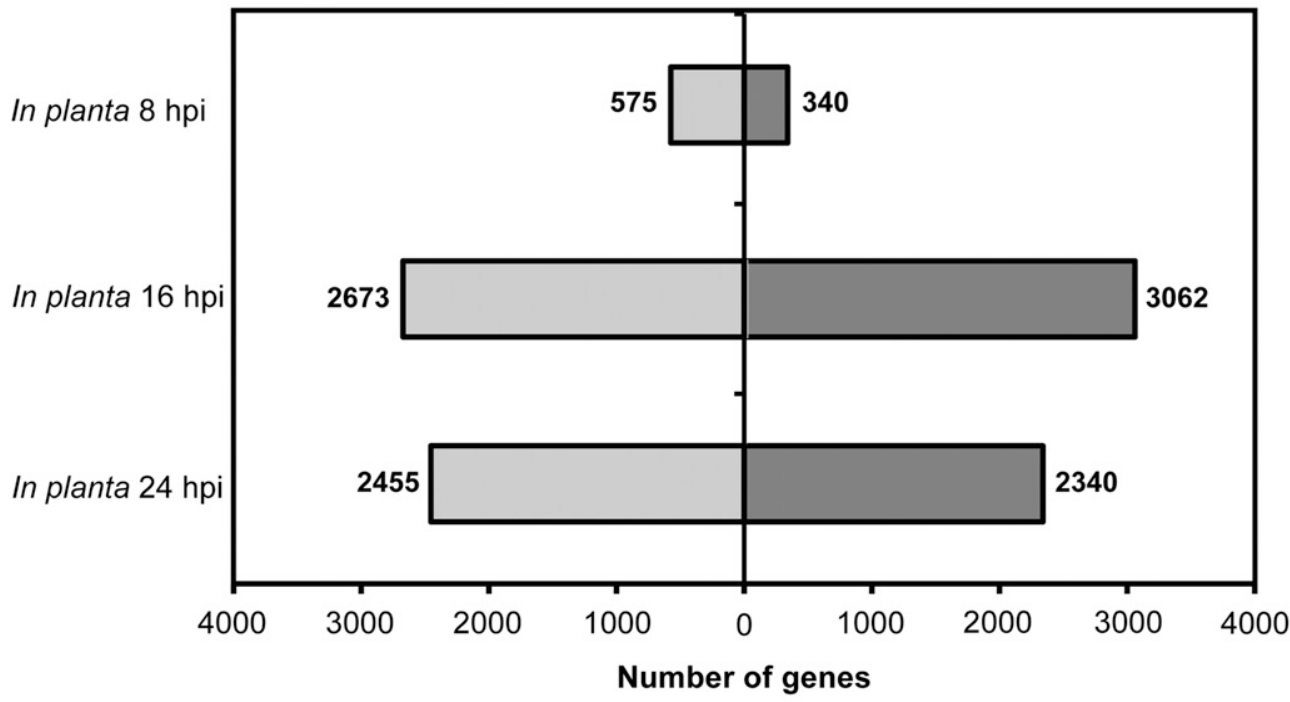

B

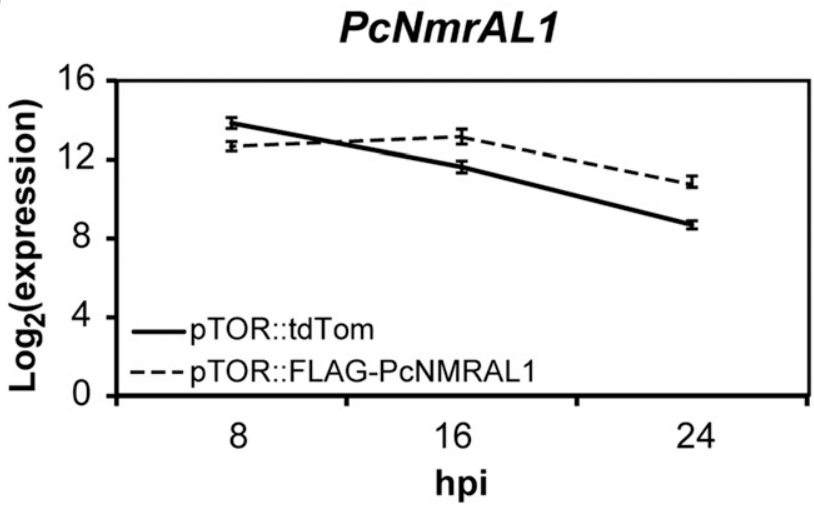

D

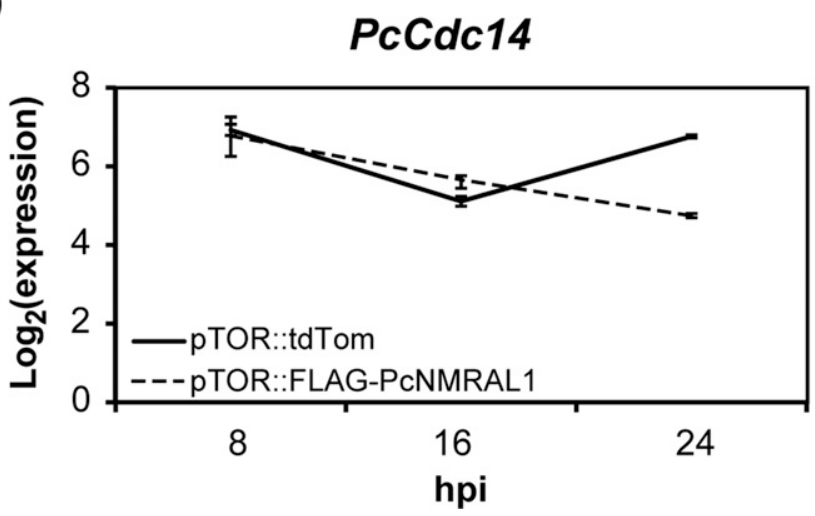

C

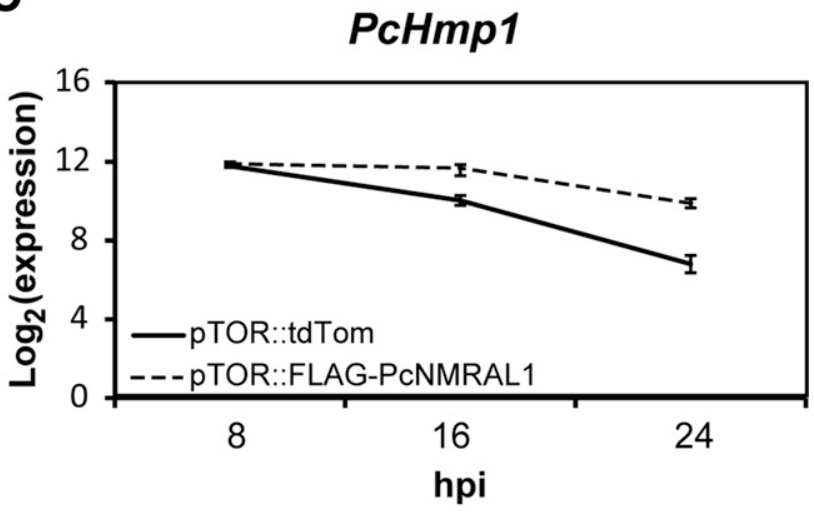

E

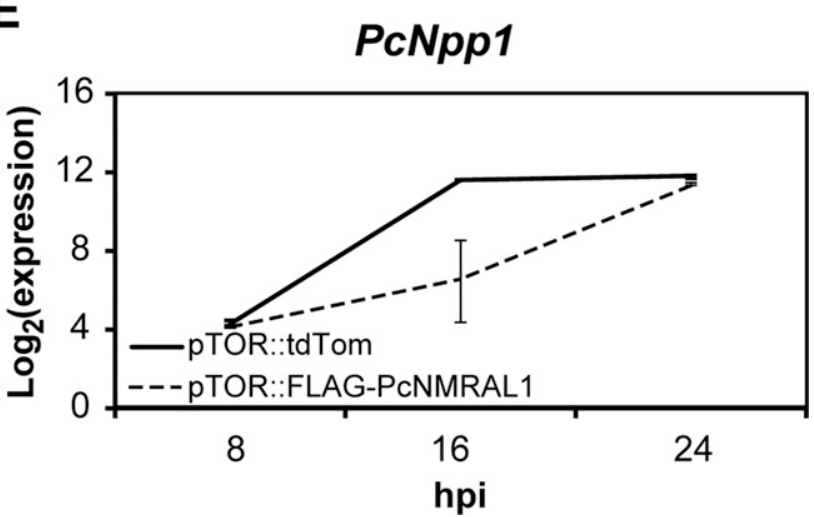

Fig. 4. Overexpression of $P c N m r A L 1$ results in both induction and repression of gene expression and affects expression of infection-related marker genes. A, Number of genes that were significantly more up- or down-regulated in the pTOR::FLAG-NMRAL1 line compared with the pTOR::tdTom line in planta at each timepoint during the time course microarray experiment. The number of genes represented by each bar are shown next to the bars. B, Microarray expression patterns of PcNmrAL1, C, PcHmpl (Phyca11_8692), D, PcCdc14 (Phyca11_510939), and E, PcNpp1 (Phyca11_11951) in pTOR::tdTom and pTOR::FLAG-PcNMRAL1 lines over the time course microarray experiment. 
infection process by extending the biotrophic phase or delaying the onset of necrotrophy or both.

\section{Differences in gene expression patterns are linked to PcNMRALI overexpression and not solely due} to altered growth in the pTOR::FLAG-PcNMRAL1 line.

Overexpression of PcNmrALI leads to a virulence phenotype with slower lesion growth and reduced biomass production on tomato (Fig. 2). It is, therefore, possible that the observed differences in gene expression are due to either the sampling of dissimilar infection stages caused by the reduced growth rate or the observed reduction in biomass for our pTOR::FLAGPcNMRAL1 line. To exclude these possibilities and to further test whether the changes in gene expression seen in the pTOR:: FLAG-PcNMRAL1 line during infection is due to PcNmrAL1 overexpression, we used microarrays and subsequent DEG analyses to measure, quantify, and compare gene expression between our strains in mycelia grown in vitro for $24 \mathrm{~h}$. These analyses revealed 1,907 DEGs, 948 of which were up-regulated and 959 were down-regulated as a consequence of PcNmrAL1 overexpression (Supplementary File S2). Direct comparisons of gene lists emanating from our in-vitro and in-planta experiments revealed 85 and $73 \%$ of genes found upregulated in vitro, were also found up-regulated at 16 and 24 hpi in planta, respectively (Fig. 5A). Similarly, for the DEGs that were down-regulated in vitro upon PcNmrAL1 overexpression, 71 and $35 \%$ overlapped with those downregulated in planta at the 16- and 24-hpi timepoints, respectively (Fig. 5A). These results contrasted with comparisons of DEGs identified at the 8-h timepoint as only $2 \%$ (upregulated) and 6\% (downregulated) of the DEGs identified in vitro were common with DEGs identified at $8 \mathrm{~h}$ (Fig. 5A). These results support the notion $\mathrm{i}$ ) that transcriptional changes induced by PcNMRAL1 are genuine and not due to sampling of distinct life stages, and thus, ii) PcNMRAL1 is a TR, specifying the expression of $P$. capsici genes during the early stages of infection, and iii) the regulatory activity of PcNMRAL1 toward its downstream targets and the consequent changes in their expression may underpin the observed extension of biotrophy and delay of necrotrophy.

Previous work in our lab identified 57, 209, and 532 genes as coexpressed with $P c H m p 1, P c N p p 1$, and $P c C d c 14$, respectively (Jupe et al. 2013). Given the significant overlap between genes differentially regulated both in planta and in vitro and the observed changes in infection, we assessed to what extent the expression of stage-specific genes, as defined by Jupe et al. (2013), are affected by $P c N m r A L 1$ overexpression in vitro. Direct comparisons of genes coexpressed with PcHmpl (biotrophy), PcNppl (necrotrophy), and $P c C d c 14$ (sporulation) against $P c N m r A L 1$-regulated genes in vitro, revealed that $26.7 \%$ of biotrophy-associated genes are induced upon PcNmrALl overexpression in vitro, whereas $38.6 \%$ of $P c N p p 1$-coexpressed genes are repressed in our in-vitro experiment (Fig. 5B). These results contrast with those found for $P c C c d 14$-coexpressed genes, whose expression does not appear to be affected by PcNmrAL1 (Fig. 5B). Given that few biotrophyassociated genes appear to be downregulated in vitro and, conversely, a small percentage of $P c N p p 1$-coexpressed genes are induced in these experiments, we surmise that PcNmrAL1 extends the biotrophic phase by both maintaining the expression of biotrophy-associated genes and reducing the levels of transcripts encoding factors required for necrotrophy.

To gain a more general overview of the biological processes affected by PcNmrAL1 overexpression that could drive prolonged biotrophy, GO analysis for significantly enriched ontologies was performed on genes that showed a significantly greater increase in expression in the pTOR::FLAG-PcNMRAL1 line and which were common between the in-vitro and in-planta stages (Supplementary Tables S3, S4, and S5). No significant GO categories were found at
8 hpi. Significant GO categories found at 16 and 24 hpi were very similar and in support of the hypothesized function of PcNMRAL1 in transcriptional regulation, as over-representation of genes involved in gene expression (GO:0010467), transcription (GO:0006350), and translation (GO:0006412) were revealed. Interestingly, genes involved in nitrogen compound metabolic processes (GO:006807) were also enriched for, in addition to several other metabolic and biosynthetic processes (Fig. 6). Conversely, in terms of genes that were found to be significantly downregulated in the pTOR:: FLAG-PcNMRAL1 line and common between the in-vitro and inplanta stages (Supplementary Tables S6 and S7), only one GO term, carbohydrate metabolic process (GO:0005975), was found to be over-represented at $16 \mathrm{hpi}$, with no significant terms found at 24 hpi. Overall, our results point toward a role of PcNMRAL1 in the regulation of gene expression.

\section{Expression of TFs and regulators are affected by PcNMRAL1.}

Given the large transcriptional changes seen in $P$. capsici during infection upon overexpression of PcNmrAL1, we expected to find TFs and TRs among the DEGs. Our analysis revealed a total of 102 TFs and TRs with altered expression between the pTOR::tdTom and pTOR::FLAG-PcNMRAL1 lines, with the largest difference seen at 16 hpi (Fig. 7A). At this timepoint, 85 and 37 TFs and TRs were up- and down-regulated, respectively (Supplementary File S3). The differences seen in the total number of DEGs and the number of TFs and TRs within these suggest overexpression of PcNmrALl has a significant impact on gene expression in $P$. capsici mediated by a number of TFs and TRs.

\section{PcNmrAL1 overexpression alters expression of effector genes.}

The observed reduction in the sizes of lesions caused by $P$. capsici lines overexpressing PcNmrAL1 together with the changes in the expression patterns of a large number of genes, including marker genes for different stages of infection, suggests expression of infection-related genes may also be altered. To investigate this possibility, we looked at the number of RxLR and crinkler (CRN) effectors within the lists of DEGs at each timepoint. This analysis identified 170 of 508 RxLR effectors and 67 of 234 CRN effectors as DEGs over the time course experiment. The trend in the number of RxLR and CRN effectors identified as differing in expression between the PcNmrALl overexpression and control lines at each timepoint was very similar to that seen with TFs and TRs, with the largest difference between the two lines seen at 16 and 24 hpi (Fig. 7B; Supplementary File S4).

Interestingly, one of the CRN effectors identified as a DEG was CRN83_152, which was previously shown to enhance $P$. capsici lesion growth when transiently expressed in Nicotiana benthamiana (Mafurah et al. 2015; Stam et al. 2013). In the pTOR::tdTom line, CRN83_152 expression increases between 8 and $16 \mathrm{hpi}$, with the level of expression at $24 \mathrm{hpi}$ similar to that at 16 hpi; in the PcNmrAL1 overexpression line, CRN83_152 expression was significantly lower at $16 \mathrm{hpi}$ compared with the control line (Fig. 7C). Another effector identified as a DEG was PcRxLR516, a homolog of the P. infestans RxLR Pi03192 (PITG_03192), which targets the $N$-acetyl-l-cysteine TFs NPT1 and NPT2 during infection and is required for full pathogenicity (McLellan et al. 2013). Whereas PcRxLR516 expression is highest at 8 hpi and decreases over the duration of the experiment in the control line, expression of this effector does not decrease until after 16 hpi in the pTOR::FLAG-PcNMRAL1 line (Fig. 7D).

\section{DISCUSSION}

P. capsici is a broad host-range pathogen that is able to infect and cause disease on many economically important crop plants. 
As such, identifying key regulators that drive the infection process could lead to novel targets, suited for chemical control of $P$. capsici and related pathogens on a wide range of host plants. Our previous work highlighted dramatic transcriptional changes during $P$. capsici infection, which has led to the suggestion that its regulation is required for a parasitic lifestyle (Jupe et al. 2013). If true, perturbing these molecular events may disrupt or hamper infection-associated processes, thereby leading to attenuation in virulence. Here, we identified and characterized a candidate TR called PcNMRAL1. PcNMRAL1 (Phyca11_505845) is related to AnNMRA in A. nidulans and features an expression pattern that is similar to PcHmpl, a marker of biotrophy in $P$. capsici. In fungi, such as $A$. nidulans and Neurospora crassa, the function of NMRA-like proteins has been well-studied and have been shown to act as TRs involved in nitrogen metabolite repression (Andrianopoulos et al. 1998; DeBusk and Ogilvie 1984; Kotaka et al. 2008; Lamb et al. 2004; Stammers et al. 2001; Xiao et al. 1995). NMRA-like proteins have also been identified in organisms other than fungi, such as the social amoeba Dictyostelium discoideum, in which NMRA-like protein PadA is proposed to act as a positive TR of cAMP-induced genes (Garciandia and Suarez 2013; Núñez-Corcuera et al. 2008). Given the roles of NMRA-like proteins in transcriptional regulation in other organisms, we hypothesized that PcNMRAL1 may be an important factor driving $P$. capsici infection.

Here, we present evidence that PcNMRAL1 fulfils an important function required for infection. Overexpression of PCNmrALl in $P$. capsici resulted in reduced colonization of the host, evidenced by a lower rate of lesion growth on tomato leaves and a dramatic reduction in biomass during infection. In addition to a visible virulence phenotype, qRT-PCR and microarray analyses revealed altered expression of $\mathrm{PcHmpl}$ and $\mathrm{PcNppl}$, key infection marker genes reporting on the biotrophic and necrotrophic infection
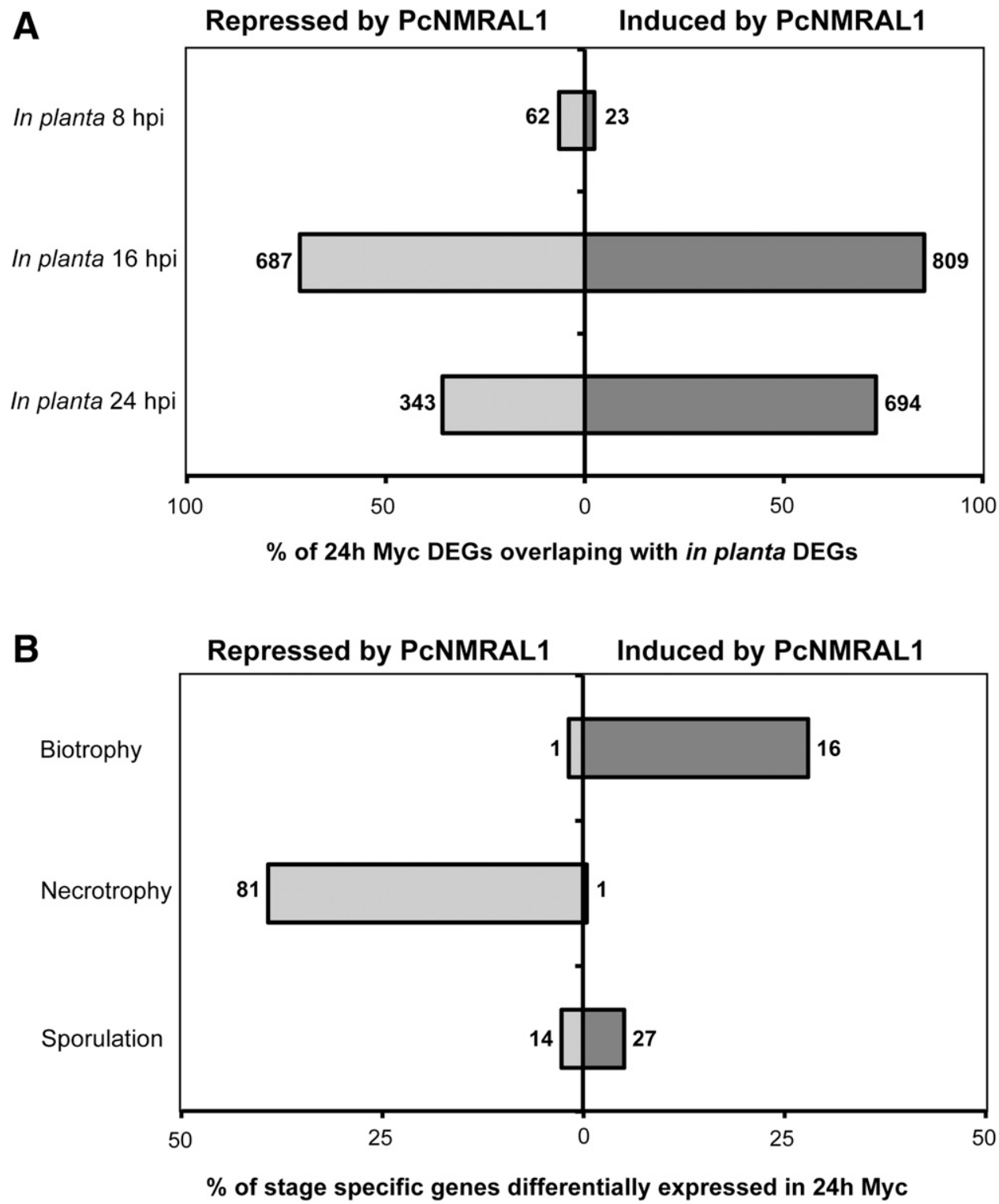

Fig. 5. Overexpression of PcNmrALl affects a common set of genes both in planta and in vitro and suggests prolonged biotrophy or repressed necrotrophy in planta. A, Overlap of differentially expressed genes (DEGs) identified by microarray analysis in vitro (mycelia grown in liquid media for $24 \mathrm{~h}$ ) and in planta during a time course infection assay. The number of common DEGs (i.e., genes significantly up- or downregulated in the pTOR::FLAG-PcNMRAL1 line compared with the pTOR::tdTom line) between the in vitro and in planta stages are presented as a percentage of the total number of DEGs at the mycelial stage grown in vitro. B Percentage of biotrophy $(n=58)$, necrotrophy $(n=210)$, and sporulation $(n=533)$ genes induced or repressed by PcNMRAL1 grown in vitro. Genes associated with the specific stages of infection were as described by Jupe et al. (2013). Figures next to bars are the number of genes represented by the bars. 
phases, respectively, suggesting extension of biotrophy and a delay in the onset of necrotrophy. Consistent with a role in gene regulation and infection, we identified a substantial number of genes that may be direct or indirect targets of PcNMRAL1. Microarray analysis of infection time courses for pTOR::tdTom and the pTOR::FLAG-PcNMRAL1 lines revealed that PcNmrAL1 overexpression leads to substantial changes in gene expression at the later stages of infection, when endogenous PcNmrAL1 expression is normally down-regulated. By performing equivalent experiments on our strains grown in vitro, we were able to demonstrate that for the majority of possible target genes, differential gene expression seen in planta was not caused by a reduction in growth rate but was due to PcNmrAL1 overexpression. These results reiterate a role for PcNMRAL1 in gene regulation and suggest that, by controlling transcriptional programs, this protein drives developmental processes required for disease progression. Strong support for this hypothesis emanates from the observation that PcNmrALl overexpression leads to the induction of biotrophyassociated genes while suppressing necrotrophy-associated genes in mycelia grown in vitro. These dual activities explain the observed reduction in virulence and extension of the biotrophic phase.

As the DEGs identified at 16 and $24 \mathrm{hpi}$ are potentially PcNMRAL1-regulated genes, we performed GO analysis for enriched ontologies to identify the biological processes regulated by PcNMRAL1. This analysis revealed an over-representation of ontologies relating to gene expression (GO:0010467), transcription (GO:0006350), and translation (GO:0006412). This is in agreement with the large number of TFs and TRs identified as DEGs at these timepoints, suggesting that PcNMRAL1 modulates transcription of infection-related genes by regulating the expression of other TFs and TRs. Interestingly, a number of ontologies related to nitrogen compound metabolic processes (GO:006807) and other metabolic and biosynthetic processes were also over-represented in our analyses, suggesting that, by modifying the expression of metabolic genes, PcNMRAL1 integrates pathogen growth and development in response to external cues. These results would be consistent with observations made in plant-pathogenic fungi, in which nitrogen metabolism has been linked to the regulation of infection-related genes. For example, in Magnaporthe oryzae, the causal agent of rice blast, three NMRA-like proteins, namely, NMR1, NMR2, and NMR3, have been shown to act as transcriptional corepressors of pathogenicity (Wilson et al. 2010, 2007). It is hypothesized that, in response to the change in nutrient availability in planta, a NADPH-dependent genetic switch is triggered in M. oryzae, involving the inactivation of NMR1, NMR2, and NMR3, leading to derepression of the essential virulence gene SPMI (a vacuolar serine protease involved in sporulation and appressorial development) and the plasma membrane protein PTHII (required for host recognition and appressorium differentiation) (DeZwaan et al. 1999; Fernandez et al. 2012; Mathioni et al. 2011; Wilson et al. 2007, 2010).

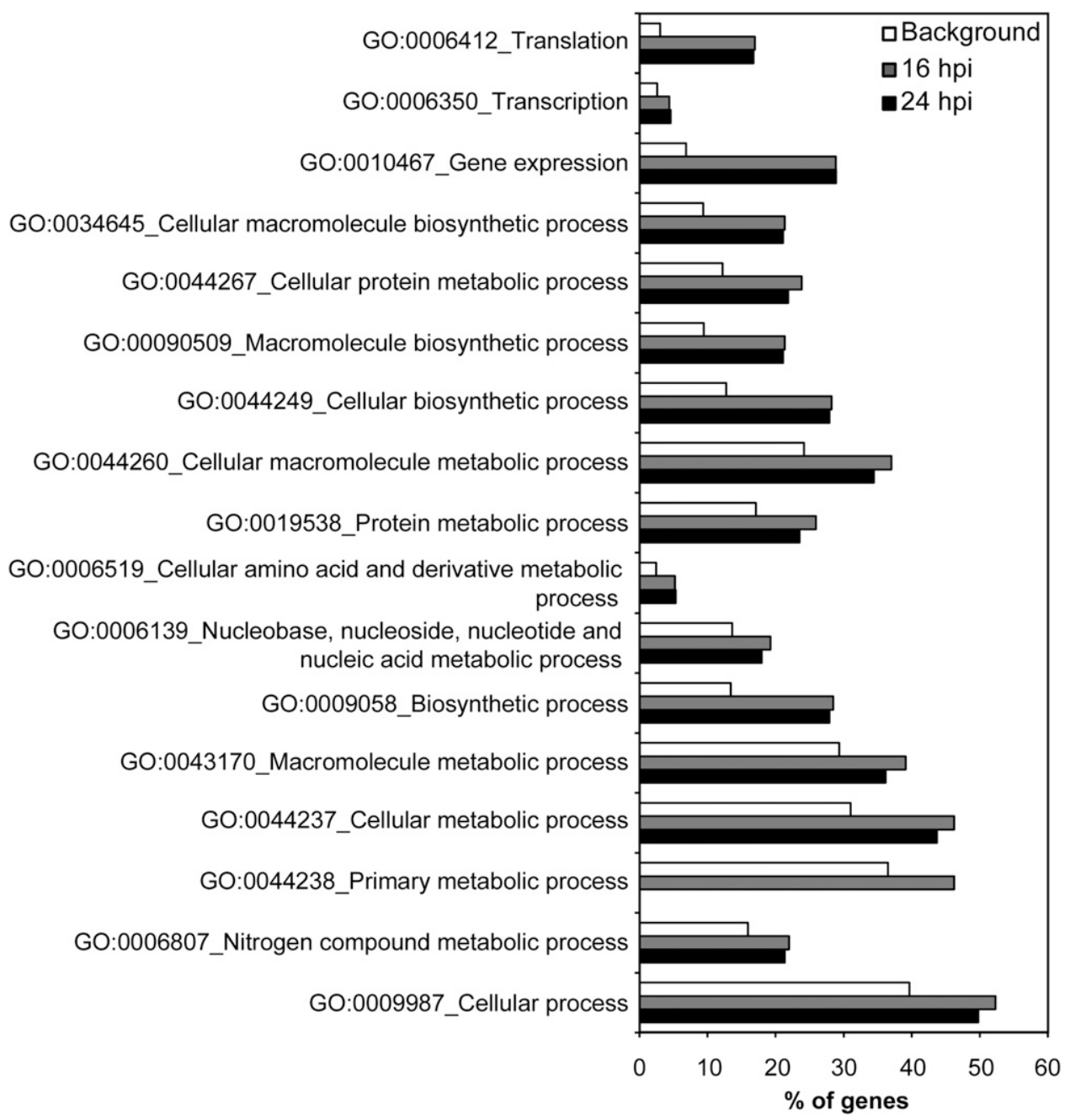

Fig. 6. Summary of ontologies significantly increased in differentially expressed genes (DEGs) shared between in-vitro (mycelia grown in liquid media for $24 \mathrm{~h}$ ) and in-planta (16 and $24 \mathrm{~h}$ postinoculation [hpi]) stages. Data shows the percentage of the DEGs identified by microarray analysis with a given ontology. Background represents the percentage of all Phytophthora capsici genes with associated gene ontology terms with the same ontology. 
Nitrogen sensing and metabolism has also been linked to virulence in Fusarium oxysporum, F. graminearum, and Cladosporium fulvum, indicating the ability to sense nitrogen availability within the host plant mediates infectious growth (López-Berges et al. 2010; Pérez-García et al. 2001). Given this link between nitrogen metabolism and pathogenicity in fungi, it is possible that PcNMRAL1 acts in a pathway linking nutritional status to the infection process in a similar manner in P. capsici. A previous study by Judelson et al. (2009) found evidence suggesting that $P$. infestans adapts its metabolism in response to nutrient availability. In addition, $P$. infestans appears to regulate the transition from biotrophy to necrotrophy by coordinated expression of effector proteins that suppress cell death, such as SNE1, and secreted proteins that induce cell death, such as PiNPP1.1 (Kelley et al. 2010; Lee and Rose 2010). This suggests Phytopthora spp. are able to sense and signal nutrient availability in planta. In our study, altered expression of several effector proteins was seen upon overexpression of PcNmrAL1, including CRN83_152 and PcRxLR516. CRN83_152 was previously shown to enhance $P$. capsici lesion growth (Stam et al. 2013), and its reduction in expression at 16 hpi in the pTOR:: PcNMRAL1 line is consistent with the reduction in lesion development observed here. PcRxLR516, a homolog of $P$. infestans Pi01392, exhibited extended expression in the PcNmrAL1 overexpression line. In P. infestans, silencing of PiO1392 was shown to reduce pathogenicity (McLellan et al. 2013). Whether or not PcRxLR516 has a similar function to Pi01392 and how the change in expression of this effector contributes to the infection phenotype observed is unknown. We hypothesize that the appropriate and coordinated expression of multiple effector proteins, in addition to their individual functions, is required for proper progression through the infection cycle and that regulators such as PcNMRAL1 play an important role in this process.

We have demonstrated that PcNMRAL1 is expressed at the earlier stages of infection, modulating the expression of factors that impact virulence, metabolism, and stage-specific gene expression. The effect of silencing or gene knockout of PcNmrAL1 on $P$. capsici virulence and gene expression in planta would provide more insight into the function of PcNMRAL1 during infection. We have been unsuccessful in silencing PcNmrAL1 in $P$. capsici and, given the very low levels of homologous recombination that occurs in Phytophthora spp., generation of targeted gene knockout mutants by classical gene disruption methods is difficult (Kamoun 2003). The use of an alternative method for targeted gene disruption using the CRISPR/Cas9 system has been successfully demonstrated in P. sojae (Fang and Tyler 2016), which we are currently optimizing for use in $P$. capsici. Successful generation of $P$. capsici strains carrying (homozygous) deleterious mutations in PcNmrAL1 should allow further assessment of PcNMRAL1 contribution toward infection.

Another critical step in understanding PcNMRAL1 function would be to define the mechanism by which PcNMRAL1 achieves gene regulation on the scale observed in our work. In fungi,
A
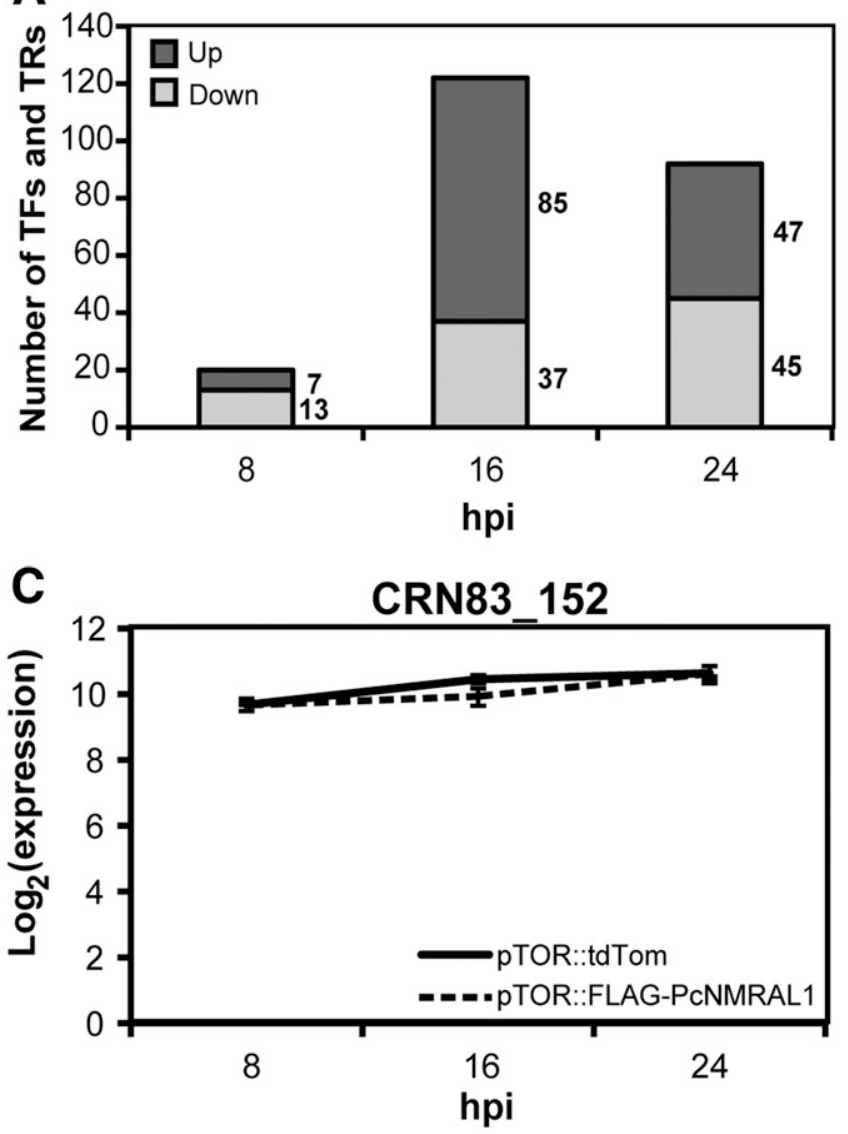

B
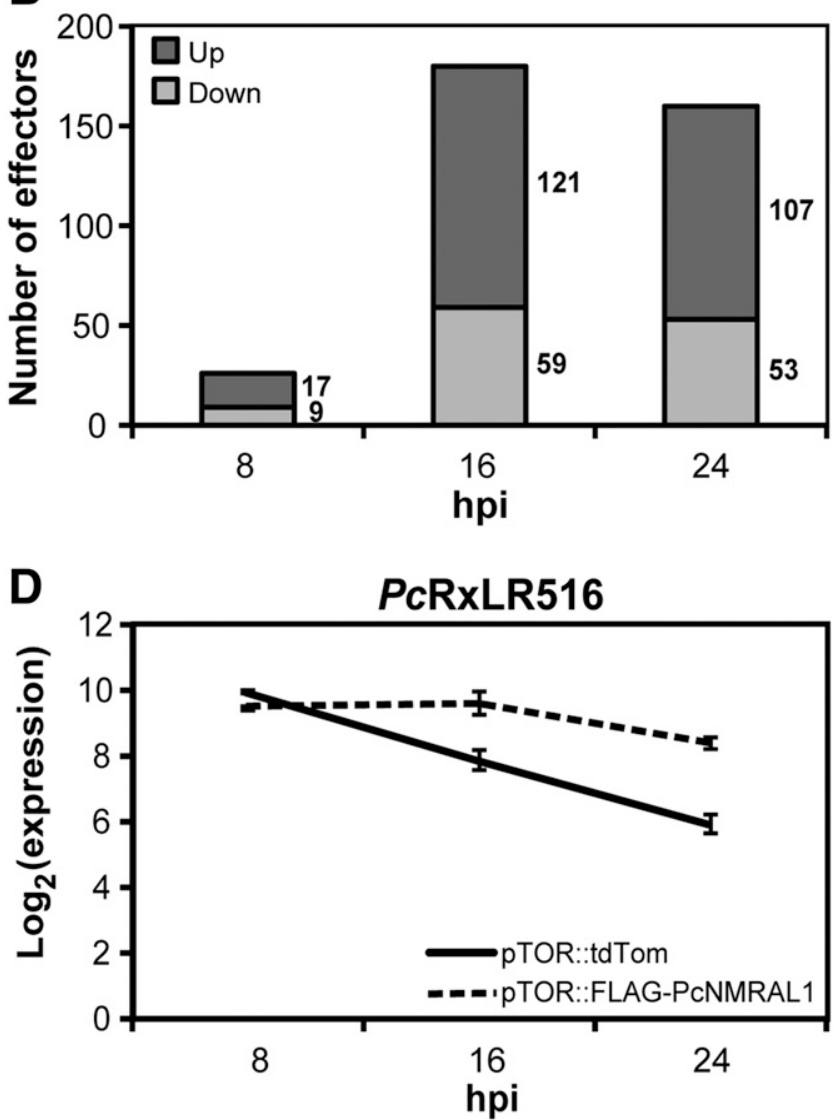

Fig. 7. Overexpression of PcNmrAL1 affects expression of many transcription factors (TFs), transcriptional regulators (TRs), and effectors. A, Data are presented as the number of TFs and TRs identified by microarray analysis as differentially expressed genes (DEGs) that were more significantly up- or downregulated in the pTOR::FLAG-PcNMRAL1 line compared with the pTOR::tdTom line. B, Number of RxLR and crinkler (CRN) effectors identified by microarray analysis as DEGs that were more significantly up- or down-regulated in the pTOR::FLAG-PcNMRAL1 line compared with the pTOR::tdTom line. Figures next to bars are the number of genes represented by the bars. C, Microarray expression patterns of CRN83_152 and D, PcRxLR516 in pTOR::tdTom and pTOR::FLAG-PcNMRAL1 lines over the time course experiment. hpi $=\mathrm{h}$ postinoculation. 
NMRA-like proteins appear to affect gene expression through physical interaction with GATA-type TFs (Andrianopoulos et al. 1998; Charoensawan et al. 2010; Kotaka et al. 2008; Lamb et al. 2004) and, in A. nidulans, expression of AnNMRA is anticorrelated with expression of the GATA-type TF AREA transcript (Wong et al. 2007). GATA-type TFs contain at least one GATA zinc finger/DNA-binding domain consisting of four cysteine residues and a basic terminal domain (Scazzocchio 2000). A PFAM search, however, failed to identify GATA-type TFs in $P$. capsici. This indicates that, although the processes regulated by PcNMRAL1 bear resemblance to those found in fungi, the mechanisms and factors that help achieve target gene regulation are likely to be different. In this context, it is worth noting that PcNMRAL1 overexpression leads to both induction and repression of $P$. capsici gene expression, suggesting engagement of PcNMRAL1 with both positive and negative regulatory factors specifying gene expression. Taken together, our results indicate that the function of NMRA-like proteins in Phytophthora spp. and any interacting partners may have diverged. In support of functional divergence of NMRA-like proteins, the NMRA-like protein PadA in Dictyostelium discoideum also appears to act as a positive regulator and is needed for full expression of the cAMP receptor CarA and normal development (Garciandia and Suarez 2013). In Cryptococcus neoformans, the NMR homolog TAR1 can act as either a positive or negative regulator, dependent on the type of nitrogen source available (Lee et al. 2012).

As PcNMRAL1 function is not likely to be mediated through interaction or modulation of a GATA-type TF, the next step would be to identify PcNMRAL1-interacting partners and the promoter binding sites of TFs regulated by PcNMRAL1. Achieving these objectives would allow us to unravel the mechanism by which this protein regulates gene expression during infection. Our data suggests that PcNMRAL1 mediates the biotrophy to necrotrophy transition and, thus, represents a novel factor that may drive the Phytophthora disease cycle on crops. This study provides a first insight into the mechanisms that regulate infection and transcription in Phytophthora spp. and provides a robust platform for further studies aimed at disabling pathogenesis and preventing crop losses.

A more thorough understanding of this and its related proteins within Phytophthora spp. or presence in other oomycete pathogens may help provide a rationale for targeting this protein and its allied processes in novel disease control strategies.

\section{MATERIALS AND METHODS}

\section{Plant material, growth of $\boldsymbol{P}$. capsici, inoculation} of leaf material, and sample collection.

P. capsici-susceptible tomato plants (S. lycoperisum cv. Moneymaker) were grown in a greenhouse at 22 to $25^{\circ} \mathrm{C}$ with 16 h of light. P. capsici strains (LT6535 and LT1534) were grown on V8 agar medium in the dark at $25^{\circ} \mathrm{C}$ for 3 days and, subsequently, at $22^{\circ} \mathrm{C}$ in the light to induce sporangia formation. To harvest sporangia and induce zoospore release, plates were flooded with ice-cold water, were agitated with a plate spreader to dislodge sporangia, and the sporangia suspension was incubated at $22^{\circ} \mathrm{C}$ in the light until motile zoospores were released. Zoospores were diluted to a concentration of $1 \times 10^{5}$ spores $\mathrm{ml}^{-1}$ and were used to inoculate 4- to 5-week-old detached tomato leaves by placing 20- $\mu$ d droplets onto the abaxial surface. Leaves were kept in a box lined with moist tissue, to create a humid environment, and were incubated at $22^{\circ} \mathrm{C}$ with a $16-\mathrm{h}$ photoperiod, for the duration of the experiment. For lesion measurements, the diameter of the lesion was measured with a ruler.

For RNA or protein extraction from infected leaf tissue, leaf discs containing the inoculation site were collected using a core borer ( $5 \mathrm{~mm}$ diameter) and were flash frozen in liquid nitrogen before extraction.

\section{Generation of tdTomato and \\ FLAG-PcNMRAL1 overexpression constructs.}

The full-length PcNmrAL1 gene (Phyca11_505845) was cloned with an N-terminal FLAG tag (DYKDDDDK) into vector pTOR (GenBank: EU257520.1). pTOR contains the ham 34 promoter and the $n p t I I$ gene conferring resistance to geneticin in $P$. capsici. Primers FLAG_PcNmrAL1_F (5' GACTACAAAGACGATGACGACAAGGTCAAGTACGTTC TCACTG-3') and PcNmrAL1_XbaI_R (5'-TGCTTATCTAG ATTACTGGTTCTTGGGGAAGATC-3') were used to amplify full-length PcNmrAL1 with the N-terminal FLAG tag (underlined) and a C-terminal $X b a \mathrm{I}$ restriction site (in italics), respectively. The PCR product was then used as template for primers ClaI_FLAG_F (5'-TAAGCAATCGATACCATGGACT ACAAAGA-3') and PcNmrAL1_XbaI_R to introduce an Nterminal ClaI site (in italics) upstream of the FLAG tag. The PCR product was then cloned between the ClaI and XbaI sites of pTOR, using the InFusion HD cloning kit (Clontech). The resulting construct was named pTOR::FLAG-PcNMRAL1. $P$. capsici strains were also transformed with a pTOR::tdTom construct that expresses the fluorescent tdTomato protein.

\section{Transformation of $\boldsymbol{P}$. capsici protoplasts.}

P. capsici strains LT1534 and LT6535 were transformed with the constructs, using a modified protoplast transformation method by Judelson et al. (1991). Sporangia collected from $P$. capsici grown on V8 agar plates, as described above, were filtered through a $60-\mu \mathrm{m}$ mesh and were used to inoculate amended lima bean broth containing ampicillin at $50 \mu \mathrm{g} \mathrm{ml}^{-1}$ and rifampicin at $10 \mu \mathrm{g} \mathrm{ml}^{-1}$. Cultures were incubated in the dark at $25^{\circ} \mathrm{C}$ overnight. Mycelia were collected and digested with, per milliliter, $5 \mathrm{mg}$ of lysing enzyme from Trichoderma harzianum and $2 \mathrm{mg}$ of cellulase from Trichoderma reesi (SigmaAldrich). Protoplasts were collected by filtering through a $30-\mu \mathrm{m}$ mesh and centrifuging at $700 \times g$ for $4 \mathrm{~min}$. Protoplasts were washed once with $\mathrm{KC}$ solution $\left(0.64 \mathrm{M} \mathrm{KCl}, 0.2 \mathrm{M} \mathrm{CaCl}_{2}\right)$, once with a 1:1 mixture of KC-MT20 solution (1 M mannitol, $20 \mathrm{mM} \mathrm{CaCl}_{2}, 10 \mathrm{mM}$ Tris-HCl, pH 7.5), and once with MT20 solution. Protoplasts were resuspended in MT10 solution (1 M mannitol, $10 \mathrm{mM} \mathrm{CaCl}_{2}$ ).

Protoplasts were added to $50 \mu \mathrm{g}$ of plasmid DNA mixed with lipofectin (Invitrogen) and were left to stand for $5 \mathrm{~min}$ before the addition of polyethylene glycol (PEG) solution $(50 \%$ [wt/vol] PEG 3350, $10 \mathrm{mM} \mathrm{CaCl}_{2}, 10 \mathrm{mM}$ Tris-HCl, $\mathrm{pH}$ 7.5). After allowing the protoplast-PEG mixture to stand for another $5 \mathrm{~min}, 10 \mathrm{ml}$ of $\mathrm{V} 8$ sucrose mannitol (V8 medium supplemented with $1 \mathrm{M}$ mannitol and $2 \%$ [wt/vol] sucrose) was added, and then, a further $12 \mathrm{ml}$ of V8 sucrose mannitol was added with ampicillin to a final concentration of $50 \mu \mathrm{g} \mathrm{ml}^{-1}$. Protoplasts were left to regenerate for $48 \mathrm{~h}$, were plated onto V8 agar media containing $50 \mu \mathrm{g}$ of geneticin per milliliter to select for transformants, and were incubated in the dark at $25^{\circ} \mathrm{C}$.

\section{Confirmation of transformants.}

Transformants carrying the pTOR::FLAG-PcNMRAL1 construct were confirmed by qRT-PCR and Western blot. Material for both assays was generated from mycelial tissue grown in pea broth for 2 days in the dark. Overexpression of PcNmrAL1 was confirmed by qRT-PCR, as described below.

Proteins were extracted in GTEN buffer $(10 \%$ [vol/vol] glycerol, $25 \mathrm{mM}$ Tris-HCl, pH 7.5, $1 \mathrm{mM}$ EDTA, $150 \mathrm{mM} \mathrm{NaCl}$ ), supplemented with $2 \%$ polyvinylpolypyrrolidone, $10 \mathrm{mM}$ dithiothreitol, and $1 \times$ protease inhibitor, and were separated by sodium dodecyl sulfate-polyacrylamide gel electrophoresis. Proteins were 
then transferred to $0.2-\mu \mathrm{M}$ polyvinylidene diflouride membrane (Bio-Rad). Expression of FLAG-PcNMRAL1 protein was confirmed by Western blot probed with Oct-A probe conjugated with horseradish peroxidase raised in mouse against the FLAG tag (Santa Cruz Biotechnology) and was visualized by chemiluminescence using SuperSignal West Femto maximum sensitivity substrate (Thermo Scientific).

\section{qRT-PCR and qPCR.}

For quantification of gene expression, RNA was extracted using an RNeasy kit (Qiagen) following manufacturer's instructions, and cDNA was generated by reverse transcription, using Super Script III reverse transcriptase (Invitrogen) with Oligo(dT) 15 primer (Promega). qRT-PCR was performed using FastStart universal probe master (Rox) (Roche). Gene expression levels were calculated relative to either expression in the control line or at the first timepoint, using the cycle threshold $\left(\Delta \Delta \mathrm{C}_{\mathrm{T}}\right)$ method. Primers used for detection of PcNmrALI were PcNmrAL1-F: 5'-GAGGAGCTCCCGTTCCTG-3' and PcNmrAL1-R: 5'-CCAGACCGGAAGCGTAGA-3' with UPL probe \#131. Expression levels of PcNmrAL1 were normalized against $P$. capsici tubulin. Primers used for detection of $P$. capsici tubulin (Phyca11_576734) were PcTub-F: 5'-GCAATACCAGG ATGCTACCG-3' and PcTub-R: 5'-CATCTCGTCCATCTCCT CGT-3' with UPL probe \#63.

For quantification of $P$. capsici biomass in planta, tomato leaves were infected with 10- $\mu$ l droplets of $P$. capsici zoospores as described above. Leaf discs, $10 \mathrm{~mm}$ in diameter, containing the inoculation site and surrounding tissue were harvested at 16 hpi with a core borer; each biological replicate consisted of four leaf discs from three leaves, for a total of 12 leaf discs per sample. DNA was extracted using Qiagen DNeasy kit following manufacturer's instructions. The ratio of $P$. capsici tubulin detected against tomato tubulin was used as a measure of P. capsici biomass. Tomato tubulin (Solyc08g006890.2.1) was detected using primers TomTub_F: 5'-CATGGCTTGCTGTC TCATGT-3' and TomTub_R: 5'-CCACAGCAGCATTAACA TCC-3' with UPL probe \#9.

\section{Statistical analysis.}

Statistical significance of differences in lesion sizes and gene expression as measured by qRT-PCR were determined using the R packages nlme (Pinheiro et al. 2016) for linear effects models and multcomp (Hothorn et al. 2008) or lsmeans (Lenth 2016) to calculate Tukey contrasts.

\section{Microarray of time course infection assays and in vitro tissue.}

For in planta tissue, tomato leaves were inoculated with $P$. capsici zoospores (LT6535 background) and leaf discs collected as described above at 8,16 , and 24 hpi. Each biological replicate consisted of a total of eight leaf discs collected from two leaves.

For in vitro tissue, pea broth was inoculated with $P$. capsici zoospores and was collected after $24 \mathrm{~h}$ of growth in the dark. Experiments were repeated three times to give three biological replicates for each sample. RNA was extracted from samples using Qiagen RNeasy RNA extraction kit, following manufacturer's instructions. The microarray experiment was performed using customized $8 \times 60 \mathrm{k}$ Agilent array slides (details below). The predicted transcripts of $P$. capsici (LT1534 v11) (Lamour et al. 2012a), supplemented with 234 CRN and 508 RxLR effectors predicted in-house (Lamour et al. 2012b; Stam et al. 2013), were used to generate probe sets with Agilent eArray software. Redundant genes were replaced using in house-generated Python scripts. Four copies of each probe were hybridized to each array as described by Jupe et al. (2013).

\section{Analysis of microarray data.}

Microarray data were quantile normalized between all arrays, and DEG analysis was carried out using the R package Limma (Ritchie et al. 2015; Smyth 2004). Raw data are available at ArrayExpress (accession E-MTAB-5620). Between-array weights were calculated using arrayWeights (Ritchie et al. 2006) and between duplicate spots correlation were calculated using dupcor (Smyth et al. 2005). A total of $25 \%$ of the lowest expressed probes were removed before DEG analysis, as below. Within each $P$. capsici line, genes were classed as differentially expressed if expression of a gene significantly changed between at least two of the timepoints sampled. This was determined using lmFit and eBayes with Benjamani and Hochberg multiple testing correction $(P<0.005)$. These DEGs were used to look for coregulated genes in each line, using Pearson correlation, as described below.

Genes differentially expressed in the pTOR::FLAG-PcNMRAL1 line compared with the pTOR::tdTom line were also determined, using $\mathrm{lmFit}$ and eBayes with Benjamani and Hochberg multiple testing correction $(P<0.005)$. In this case, genes were classed as differentially expressed at a timepoint if it was significantly more up- or down-regulated in the pTOR::FLAG-PcNMRAL1 line compared with the control pTOR::tdTom line.

To look for genes coregulated with a specified marker gene in each line, similarities in gene expression profiles of DEGs were calculated using Pearson correlation in $\mathrm{R}$, and expression data was extracted based on a minimum Pearson correlation of 0.95 .

GO analysis of DEGs for over-represented biological processes was carried out using agriGO with a singular enrichment strategy (Du et al. 2010). Significance was tested using Fisher's exact test at $P<0.05$, with Benjamini and Hochberg multiple correction, and results were reported with generic GO slim.

\section{Data access.}

The microarray data generated during the current study are available in the ArrayExpress depository under accession EMTAB-5620 and the experimental design and probe sequences under A-MTAB-610. Scripts used for normalization and DEG analysis can be found at GitHub.

\section{ACKNOWLEDGMENTS}

P. capsici cultures were held at The James Hutton Institute under license PH\6 2015 . We thank S. C. Whisson for comments, suggestions, and advising on Phytophthora transformation procedures, and P. R. J. Birch for comments and suggestions. We also thank P. E. Hedley and J. A. Morris for microarray technical assistance and G. J. A. Thilliez for assistance with coexpression analysis.

\section{LITERATURE CITED}

Ah Fong, A. M. V., and Judelson, H. S. 2003. Cell cycle regulator Cdc14 is expressed during sporulation but not hyphal growth in the fungus-like oomycete Phytophthora infestans. Mol. Microbiol. 50:487-494.

Andrianopoulos, A., Kourambas, S., Sharp, J. A., Davis, M. A., and Hynes, M. J. 1998. Characterization of the Aspergillus nidulans nmrA gene involved in nitrogen metabolite repression. J. Bacteriol. 180:1973-1977.

Avrova, A. O., Boevink, P. C., Young, V., Grenville-Briggs, L. J., van West, P., Birch, P. R. J., and Whisson, S. C. 2008. A novel Phytophthora infestans haustorium-specific membrane protein is required for infection of potato. Cell. Microbiol. 10:2271-2284.

Birch, P. R. J., and Whisson, S. C. 2001. Phytophthora infestans enters the genomics era. Mol. Plant Pathol. 2:257-263.

Charoensawan, V., Wilson, D., and Teichmann, S. A. 2010. Genomic repertoires of DNA-binding transcription factors across the tree of life. Nucleic Acids Res. 38:7364-7377.

DeBusk, R. M., and Ogilvie, S. 1984. Nitrogen regulation of amino acid utilization by Neurospora crassa. J. Bacteriol. 160:493-498.

DeZwaan, T. M., Carroll, A. M., Valent, B., and Sweigard, J. A. 1999. Magnaporthe grisea pth $11 \mathrm{p}$ is a novel plasma membrane protein that 
mediates appressorium differentiation in response to inductive substrate cues. Plant Cell 11:2013-2030.

Du, Z., Zhou, X., Ling, Y., Zhang, Z., and Su, Z. 2010. agriGO: A GO analysis toolkit for the agricultural community. Nucleic Acids Res. 38 (suppl_2):W64-W70.

Dunn-Coleman, N. S., Tomsett, A. B., and Garrett, R. H. 1981. The regulation of nitrate assimilation in Neurospora crassa: Biochemical analysis of the $n m r-1$ mutants. Mol. Gen. Genet. 182:234-239.

Fang, Y., and Tyler, B. M. 2016. Efficient disruption and replacement of an effector gene in the oomycete Phytophthora sojae using CRISPR/Cas9. Mol. Plant Pathol. 17:127-139.

Fernandez, J., Wright, J. D., Hartline, D., Quispe, C. F., Madayiputhiya, N., and Wilson, R. A. 2012. Principles of carbon catabolite repression in the rice blast fungus: Tps1, Nmr1-3, and a MATE-family pump regulate glucose metabolism during infection. PLoS Genet. 8:e1002673.

Garciandia, A., and Suarez, T. 2013. The NMRA/NMRAL1 homologue PadA modulates the expression of extracellular cAMP relay genes during aggregation in Dictyostelium discoideum. Dev. Biol. 381:411-422.

Haas, B. J., Kamoun, S., Zody, M. C., Jiang, R. H. Y., Handsaker, R. E., Cano, L. M., Grabherr, M., Kodira, C. D., Raffaele, S., Torto-Alalibo, T., Bozkurt, T. O., Ah-Fong, A. M. V., Alvarado, L., Anderson, V. L., Armstrong, M. R., Avrova, A., Baxter, L., Beynon, J., Boevink, P. C., Bollmann, S. R., Bos, J. I. B., Bulone, V., Cai, G., Cakir, C., Carrington, J. C., Chawner, M., Conti, L., Costanzo, S., Ewan, R., Fahlgren, N., Fischbach, M. A., Fugelstad, J., Gilroy, E. M., Gnerre, S., Green, P. J., Grenville-Briggs, L. J., Griffith, J., Grünwald, N. J., Horn, K., Horner, N. R., Hu, C. H., Huitema, E., Jeong, D. H., Jones, A. M., Jones, J. D., Jones, R. W., Karlsson, E. K., Kunjeti, S. G., Lamour, K., Liu, Z., Ma, L., Maclean, D., Chibucos, M. C., McDonald, H., McWalters, J., Meijer, H. J., Morgan, W., Morris, P. F., Munro, C. A., O’Neill, K., OspinaGiraldo, M., Pinzón, A., Pritchard, L., Ramsahoye, B., Ren, Q., Restrepo, S., Roy, S., Sadanandom, A., Savidor, A., Schornack, S., Schwartz, D. C., Schumann, U. D., Schwessinger, B., Seyer, L., Sharpe, T., Silvar, C., Song, J., Studholme, D. J., Sykes, S., Thines, M., van de Vondervoort, P. J., Phuntumart, V., Wawra, S., Weide, R., Win, J., Young, C., Zhou, S., Fry, W., Meyers, B. C., van West, P., Ristaino, J., Govers, F., Birch, P. R., Whisson, S. C., Judelson, H. S., and Nusbaum, C. 2009. Genome sequence and analysis of the Irish potato famine pathogen Phytophthora infestans. Nature 461:393-398.

Hothorn, T., Bretz, F., and Westfall, P. 2008. Simultaneous inference in general parametric models. Biom. J. 50:346-363.

Judelson, H. S., Tani, S., and Narayan, R. D. 2009. Metabolic adaptation of Phytophthora infestans during growth on leaves, tubers and artificial media. Mol. Plant Pathol. 10:843-855.

Judelson, H. S., Tyler, B. M., and Michelmore, R. W. 1991. Transformation of the oomycete pathogen Phytophthora infestans. Mol. Plant-Microbe Interact. 4:602-607.

Jupe, J., Stam, R., Howden, A. J. M., Morris, J. A., Zhang, R., Hedley, P. E., and Huitema, E. 2013. Phytophthora capsici-tomato interaction features dramatic shifts in gene expression associated with a hemi-biotrophic lifestyle. Genome Biol. 14:R63.

Kamoun, S. 2003. Mol. Genet. Pathogenic Oomycetes. 2:191-199.

Kamoun, S., Furzer, O., Jones, J. D. G., Judelson, H. S., Ali, G. S., Dalio, R. J. D., Roy, S. G., Schena, L., Zambounis, A., Panabières, F., Cahill, D., Ruocco, M., Figueiredo, A., Chen, X. R., Hulvey, J., Stam, R., Lamour, K., Gijzen, M., Tyler, B. M., Grünwald, N. J., Mukhtar, M. S., Tomé, D. F. A., Tör, M., Van Den Ackerveken, G., McDowell, J., Daayf, F., Fry, W. E., Lindqvist-Kreuze, H., Meijer, H. J. G., Petre, B., Ristaino, J., Yoshida, K., Birch, P. R. J., and Govers, F. 2015. The top 10 oomycete pathogens in molecular plant pathology. Mol. Plant Pathol. 16:413-434.

Kanneganti, T. D., Huitema, E., Cakir, C., and Kamoun, S. 2006. Synergistic interactions of the plant cell death pathways induced by Phytophthora infestans Nepl-like protein PiNPP1.1 and INF1 elicitin. Mol. Plant-Microbe Interact. 19:854-863.

Kelley, B. S., Lee, S. J., Damasceno, C. M. B., Chakravarthy, S., Kim, B. D., Martin, G. B., and Rose, J. K. C. 2010. A secreted effector protein (SNE1) from Phytophthora infestans is a broadly acting suppressor of programmed cell death. Plant J. 62:357-366.

Kotaka, M., Johnson, C., Lamb, H. K., Hawkins, A. R., Ren, J., and Stammers, D. K. 2008. Structural analysis of the recognition of the negative regulator NmrA and DNA by the zinc finger from the GATAtype transcription factor AreA. J. Mol. Biol. 381:373-382.

Lamb, H. K., Leslie, K., Dodds, A. L., Nutley, M., Cooper, A., Johnson, C. Thompson, P., Stammers, D. K., and Hawkins, A. R. 2003. The negative transcriptional regulator $\mathrm{NmrA}$ discriminates between oxidized and reduced dinucleotides. J. Biol. Chem. 278:32107-32114.

Lamb, H. K., Ren, J., Park, A., Johnson, C., Leslie, K., Cocklin, S., Thompson, P., Mee, C., Cooper, A., Stammers, D. K., and Hawkins,
A. R. 2004. Modulation of the ligand binding properties of the transcription repressor NmrA by GATA-containing DNA and sitedirected mutagenesis. Protein Sci. 13:3127-3138.

Lamour, K. H., Mudge, J., Gobena, D., Hurtado-Gonzales, O. P., Schmutz, J., Kuo, A., Miller, N. A., Rice, B. J., Raffaele, S., Cano, L. M., Bharti, A. K., Donahoo, R. S., Finley, S., Huitema, E., Hulvey, J., Platt, D., Salamov, A., Savidor, A., Sharma, R., Stam, R., Storey, D., Thines, M. Win, J., Haas, B. J., Dinwiddie, D. L., Jenkins, J., Knight, J. R., Affourtit, J. P., Han, C. S., Chertkov, O., Lindquist, E. A., Detter, C., Grigoriev, I. V., Kamoun, S., and Kingsmore, S. F. 2012a. Genome sequencing and mapping reveal loss of heterozygosity as a mechanism for rapid adaptation in the vegetable pathogen Phytophthora capsici. Mol. PlantMicrobe Interact. 25:1350-1360.

Lamour, K. H., Stam, R., Jupe, J., and Huitema, E. 2012b. The oomycete broadhost-range pathogen Phytophthora capsici. Mol. Plant Pathol. 13:329-337.

Lee, I. R., Lim, J. W. C., Ormerod, K. L., Morrow, C. A., and Fraser, J. A 2012. Characterization of an Nmr homolog that modulates GATA factormediated nitrogen metabolite repression in Cryptococcus neoformans. PLoS One 7:e32585.

Lee, S. J., and Rose, J. K. C. 2010. Mediation of the transition from biotrophy to necrotrophy in hemibiotrophic plant pathogens by secreted effector proteins. Plant Signal. Behav. 5:769-772.

Lenth, R. V. 2016. Least-squares means: The R package 1smeans. J. Stat. Softw. 69:1-33.

López-Berges, M. S., Rispail, N., Prados-Rosales, R. C., and Di Pietro, A. 2010. A nitrogen response pathway regulates virulence functions in Fusarium oxysporum via the protein kinase TOR and the bZIP protein MeaB. Plant Cell 22:2459-2475.

Mafurah, J. J., Ma, H., Zhang, M., Xu, J., He, F., Ye, T., Shen, D., Chen, Y. Rajput, N. A., and Dou, D. 2015. A virulence essential CRN effector of Phytophthora capsici suppresses host defence and induces cell death in plant nucleus. PLoS One 10: e0127965.

Mathioni, S. M., Beló, A., Rizzo, C. J., Dean, R. A., and Donofrio, N. M. 2011. Transcriptome profiling of the rice blast fungus during invasive plant infection and in vitro stresses. BMC Genomics 12:49.

McLellan, H., Boevink, P. C., Armstrong, M. R., Pritchard, L., Gomez, S., Morales, J., Whisson, S. C., Beynon, J. L., and Birch, P. R. J. 2013. An RxLR effector from Phytophthora infestans prevents re-localisation of two plant NAC transcription factors from the endoplasmic reticulum to the nucleus. PLoS Pathog. 9:e1003670.

Mendgen, K., and Hahn, M. 2002. Plant infection and the establishment of fungal biotrophy. Trends Plant Sci. 7:352-356.

Nowicki, M., Foolad, M. R., Nowakowska, M., and Kozik, E. U. 2012. Potato and tomtato late blight caused by Phytopthora infestans: An overview of pathology and resistance breeding. Plant Dis. 96:4-17.

Núñez-Corcuera, B., Serafimidis, I., Arias-Palomo, E., Rivera-Calzada, A., and Suarez, T. 2008. A new protein carrying an NmrA-like domain is required for cell differentiation and development in Dictyostelium discoideum. Dev. Biol. 321:331-342.

Pais, M., Win, J., Yoshida, K., Etherington, G. J., Cano, L. M., Raffaele, S., Banfield, M. J., Jones, A., Kamoun, S., and Saunders, D. G. 2013. From pathogen genomes to host plant processes: The power of plant parasitic oomycetes. Genome Biol. 14:211.

Pérez-García, A., Snoeijers, S. S., Joosten, M. H., Goosen, T., and De Wit, P. J. 2001. Expression of the avirulence gene Avr9 of the fungal tomato pathogen Cladosporium fulvum is regulated by the global nitrogen response factor NRF1. Mol. Plant-Microbe Interact. 14:316-325.

Pinheiro, J., Bates, D., DebRoy, S., and Sarkar, D. 2016. NLME: Linear and nonlinear mixed effects models. R package version 3.1. R Foundation for tatistical Computing, Vienna.

Ritchie, M. E., Diyagama, D., Neilson, J., van Laar, R., Dobrovic, A. Holloway, A., and Smyth, G. K. 2006. Empirical array quality weights in the analysis of microarray data. BMC Bioinformatics 7:261.

Ritchie, M. E., Phipson, B., Wu, D., Hu, Y., Law, C. W., Shi, W., and Smyth, G. K. 2015. limma powers differential expression analyses for RNAsequencing and microarray studies. Nucleic Acids Res. 43:e47.

Scazzocchio, C. 2000. The fungal GATA factors. Curr. Opin. Microbiol. 3: 126-131.

Smyth, G. K. 2004. Linear models and empirical bayes methods for assessing differential expression in microarray experiments. Stat. Appl. Genet. Mol. Biol. 3:e3.

Smyth, G. K., Michaud, J., and Scott, H. S. 2005. Use of within-array replicate spots for assessing differential expression in microarray experiments. Bioinformatics 21:2067-2075.

Stam, R., Jupe, J., Howden, A. J. M., Morris, J. A., Boevink, P. C., Hedley, P. E., and Huitema, E. 2013. Identification and characterisation CRN effectors in Phytophthora capsici shows modularity and functional diversity. PLoS One 8:e59517. 
Stammers, D. K., Ren, J., Leslie, K., Nichols, C. E., Lamb, H. K., Cocklin, S., Dodds, A., and Hawkins, A. R. 2001. The structure of the negative transcriptional regulator NmrA reveals a structural superfamily which includes the short-chain dehydrogenase/reductases. EMBO J. 20:6619-6626.

Tomsett, A. B., Dunn-Coleman, N. S., and Garrett, R. H. 1981. The regulation of nitrate assimilation in Neurospora crassa: The isolation and genetic analysis of $n m r-1$ mutants. Mol. Gen. Genet. 182:229-233.

Tyler, B. M. 2007. Phytophthora sojae: Root rot pathogen of soybean and model oomycete. Mol. Plant Pathol. 8:1-8.

Wilson, R. A., Gibson, R. P., Quispe, C. F., Littlechild, J. A., and Talbot, N. J. 2010. An NADPH-dependent genetic switch regulates plant infection by the rice blast fungus. Proc. Natl. Acad. Sci. U.S.A. 107:21902-21907.

Wilson, R. A., Jenkinson, J. M., Gibson, R. P., Littlechild, J. A., Wang, Z.-Y., and Talbot, N. J. 2007. Tps1 regulates the pentose phosphate pathway, nitrogen metabolism and fungal virulence. EMBO J. 26:3673-3685.
Wong, K. H., Hynes, M. J., Todd, R. B., and Davis, M. A. 2007. Transcriptional control of nmrA by the bZIP transcription factor MeaB reveals a new level of nitrogen regulation in Aspergillus nidulans. Mol. Microbiol. 66:534-551.

Xiao, X., Fu, Y. H., and Marzluf, G. A. 1995. The negative-acting NMR regulatory protein of Neurospora crassa binds to and inhibits the DNAbinding activity of the positive-acting nitrogen regulatory protein NIT2. Biochemistry 34:8861-8868.

\section{AUTHOR-RECOMMENDED INTERNET RESOURCES}

ArrayExpress depository: http://www.ebi.ac.uk/arrayexpress GitHub database: https://github.com/JP41819/PcNmrAL1-microarray-scripts 